\title{
Le LIF agit in vivo comme facteur instructeur de l'identité chimique des neurones
}

Le type de neurotransmetteur(s) que contient un neurone est un aspect essentiel de son identité. Pour un certain nombre de neurones, on a pu démontrer que cette identité n'est pas fixée jusqu'à un stade relativement avancé du développement, et que les tissus-cibles atteints par l'axone produisent des signaux instructeurs imposant - au cours de cette période - le transmetteur adéquat. Des expériences classiques réalisées par Nicole le Douarin chez le poulet, avaient démontré la possibilité d'une conversion du phénotype de neurones sympathiques de noradrénergique à cholinergique après greffe hétérotopique dans la moelle épinière [1]. Des expériences réciproques consistant à greffer des organes-cibles normalement innervés par des neurones sympathiques cholinergiques (glandes sudoripares du coussinet plantaire du rat) dans des zones recevant uniquement une innervation noradrénergique (peau poilue), avaient permis de confirmer l'action de facteurs instructeurs [2].

On a purifié plusieurs protéines capables d'induire les neurones à «choisir" un neurotransmetteur en culture et, parmi celles-ci, le leukemia inhibitory factor (LIF) appelé un temps, également, CDF pour cholinergic differentiation factor [3]. Il restait, toutefois, à administrer la preuve de leur activité in vivo ce qui n'apparaissait pas a priori évident en raison de l'apparente redondance entre les effets de plusieurs de ces facteurs - mise en évidence en culture - dont on craignait qu'elle masque le rôle du facteur étudié. L'équipe de Richard Palmiter (Université de Washington, Seattle, USA) a utilisé pour cette démonstration une souche de souris transgéniques chez lesquelles le gène codant pour le NGF a été mis sous le contrôle d'une région promotrice du gène de l'insuline. Une libération ciblée de NGF dans le pancréas et une hyperinnervation sympathique (adrénergique) de la glande ont été observées chez ces souris [4]. Les auteurs ont tout d'abord construit une seconde souris avec, cette fois, le gène codant pour L.IF à la place du NGF et croisé les deux souris NGF et LIF (souris NFG $\times$ LIF). Ils ont par ailleurs construit des souris doublement transgéniques en co-injectant les deux constructions (souris NGF/LIF) [5]. Les résultats obtenus sont parfaitement cohérents avec l'hypothèse d'un rôle instructeur pour LIF puisque, alors que l'hyperinnervation pancréatique a persisté, le rapport entre les concentrations de choline acétyl transférase (marqueur cholinergique) et de tyrosine hydroxylase (marqueur catécholaminergique) est passé de 0,4 chez la souris Ins-NGF à 37,1 chez l'une des souris co-injectées NGF/LIF et 7,8 chez l'une des souris croisées NGF $x$ LIF, soit une augmen- tation de 20 à 100 fois. L'innervation sympathique, normalement adrénergique, a donc subi une massive instruction cholinergique.

LIF est donc le premier facteur instructeur admis en tant que tel après contrôle in vivo. On peut s'attendre à ce que, rapidement, une méthodologie du même ordre soit utilisée pour d'autres candidats, en particulier pour le CNTF dont les propriétés, en culture, sont semblables à celles de LIF.

M.P.

1. Le Douarin NM, Renaud D, Teillet MA, Le Douarin (;H. Cholinergic differentiation of presumptive adrenergic neuroblasts in interspecific chimeras after heterotopic transplantations. Proc Nad Acad Sci USA 1975 ; 72 : 728-32.

2. Schotzinger RJ, Landis SC. Acquisition of cholinergic and peptidergic properties by sympathetic innervation of rat sweat glands requires interaction with normal target. Neuron $1990 ; 5: 91-100$.

3. Yamamori T, Fukada K. Aebersold R, Korsching $\mathrm{S}$, Fann MJ, Patterson PH. The cholinergic neuronal differentiation factor from heart cells is identical to leukemia inhibitory factor. Science $1989 ; 246$ : 1412-6.

4. Edwards RH, Rutter WJ, Hanahan D. Directed expression of NGF to pancreatic $\beta$ cells in transgenic mice leads to selective hyperinnervation of the islets. Cell 1989; $58: 161-70$.

5. Bamber BA, Masters BA, Hoyle GW, Brinster RI, Palmiter RD. Leukemia inhibitory factor induces neurotransmitter switching in transgenic mice. Proc Nall Acad Sci USA 1994; 91 : 7839-43. 